ИЗВЕСТИЯ АКАДЕМИИ НАУК ЭСТОНСКОН ССР. ТОМ ХІV СЕРИЯ ФИЗИКО-МАТЕМАТИЧЕСКИХ И ТЕХНИЧЕСКИХ НАУК. 1965, N 2

\title{
О ТОЧНОСТИ МЕТОДА МЕХАНИЧЕСКИХ КВАДРАТУР ДЛЯ ОТЫСКАНИЯ СОБСТВЕННЫХ ЗНАЧЕНИЙ И СОБСТВЕННЫХ ФУНКЦИИ ИНТЕГРАЛЬНЫХ УРАВНЕНИИ
}

В настоящей статье даны оценки ошибки, возникающей при приближенном вычислении способом механических квадратур собственных значений и соответствующих собственных функций интегрального уравнения Фредгольма второго рода. До сих пор такие оценки были получены только для собственных значений - в случае эрмитова ядра [르 и нормального ядра [4]. В работе использована идея И. П. Мысовских, связанная с применением второго итерированного ядра [']. При помощи такого приема сравниваются собственные значения и соответствующие им собственные векторы двух уравнений, определенных в одном пространстве.

Рассмотрим проблему о собственных значениях для интегрального уравненияя вида

$$
\mu x(s)-\int_{0}^{1} K(s, t) x(t) d t=0 .
$$

Ядро $K(s, t)$ предполагаем непрерывным по обоим аргументам в квадрате $0 \leqslant s, t \leqslant 1$. Кроме того предполагаем. еще, что это ядро имеет по крайней мере одно собственное значение.

После замены интегрального члена квадратурной суммой интегральное уравнение (1) заменится равносильным уравнением

$$
\mu x(s)-\sum_{j=1}^{n} A_{j} K\left(s, t_{j}\right) x\left(t_{j}\right)=\mathrm{\varrho}(s),
$$

где узлы $t_{j}$ и коэффициенты $A_{j}$ - постоянные числа для данного промежутка. Соответствующий остаточный член $\varrho(s)$ выразим через погрешность использованной квадратурной формулы для второго итерированного ядра:

$$
\begin{gathered}
\varrho(s)=\mu x(s)-\sum_{j=1}^{n} A_{j} K\left(s, t_{i}\right) x\left(t_{i}\right)=\int_{0}^{1} K(s, \tau) x(\tau) d \tau-\sum_{j=1}^{n} A_{j} K\left(s, t_{j}\right) x\left(t_{j}\right)= \\
=\frac{1}{\mu} \int_{0}^{1} K(s, \tau) \int_{0}^{1} K(\tau, t) x(t) d \tau d t-\frac{1}{\mu} \sum_{j=1}^{n} A_{j} K\left(s, t_{j}\right) \int_{0}^{1} K\left(t_{j}, t\right) x(t) d t= \\
=\frac{1}{\mu} \int_{0}^{1} \varepsilon(s, t) x(t) d t,
\end{gathered}
$$


где обозначено

$$
\varepsilon(s, t)=\int_{0}^{1} K(s, \tau) K(\tau, t) d \tau-\sum_{j=1}^{n} A_{j} K\left(s, t_{j}\right) K\left(t_{j}, t\right) .
$$

При практическом решении проблемы отбрасываем остаточный член, после чего приходим к приближенному уравнению

$$
\tilde{\mu} \tilde{x}(s)-\sum_{j=1}^{n} A_{i} K\left(s, t_{j}\right) \tilde{x}\left(t_{j}\right)=0 .
$$

Положив в равенстве (3) последовательно $s=t_{i}(i=1, \ldots, n)$ и воспользовавшись в дальнейшем обозначениями $\tilde{x}\left(t_{j}\right)=\tilde{x}_{i}, \quad K\left(t_{i}, t_{i}\right)=$ $=K_{i j}, \varrho\left(t_{i}\right) \equiv \varrho_{i}$, получим систему линейных уравнений относительно неизвестных $\tilde{x}_{i}$ :

$$
\tilde{\mu} \tilde{x}_{i}-\sum_{j=1}^{n} A_{i} K_{i j} \tilde{x}_{i}=0 \quad(i=1, \ldots, n)
$$

Система (4) имеет нетривиальное решение тогда и только тогда, когда определитель системы равен нулю. Для однозначного определения найденного вектора $\tilde{x}=\left(\tilde{x}_{1}, \ldots, \tilde{x}_{n}\right)$ используем дополнительное условие $\sum_{j=1}^{n} f_{j} \tilde{x}_{j}=1$, где $f=\left(f_{1}, \ldots, f_{n}\right)-n$-мерный вектор *.

Таким образом, метод механических квадратур для приближенного вычисления искомого собственного значения $\mu$ ядра $K(s, t)$ сводится к вычислению собственного значения $\tilde{\mu}$ матрицы $\left(A_{j} K_{i j}\right)(i, j=1, \ldots, n)$. Вместо непрерывной собственной функции $x(s)$ ядра $K(s, t)$ теперь вычисляется дискретная величина - собственный вектор $\tilde{x}=\left(\tilde{x}_{1}, \ldots, \tilde{x}_{n}\right)$ упомянутой матрицы. Найденное собственное значение $\tilde{\mu}$ и соответствующий ему собственный вектор $\tilde{x}$ примем за приближенные величины собственного значения $\mu$ и $n$-мерного вектора $x=\left(x_{1}, \ldots, x_{n}\right)$, где $x_{j}=x\left(t_{j}\right)$ и вектор $x$ нормирован условием $\sum_{j=1}^{n} f_{j} x_{j}=1$.

Для оценки сделанной при этом прогрешности найдем при помощи уравнений (2) и (3), что

$$
\mu x_{i}-\tilde{\mu} \tilde{x}_{i}-\sum_{j=1}^{n} A_{j} K_{i j}\left(x_{j}-\tilde{x}_{j}\right)=\mathrm{Q}_{i} \quad(i=1, \ldots, n)
$$

с дополнительным условием нормированности $\sum_{j=1}^{n} f_{i}\left(x_{j}-\tilde{x}_{j}\right)=0$. Полученную систему перепишем в виде

- За вектор $f$ можно принять собственный вектор сопряженной матрицы $\left(A_{j} K_{i j}\right)$ * соответствующий собственному значению $\tilde{\mu}$. 


$$
\sum_{j=1}^{n} f_{j}\left(x_{j}-\tilde{x}_{j}\right)=0 .
$$

Предположим существование * обратной матрицы $\Gamma=L^{-1}=\left(\gamma_{i j}\right) \quad(i, j=1, \ldots, n, n+1)$, где обозначено

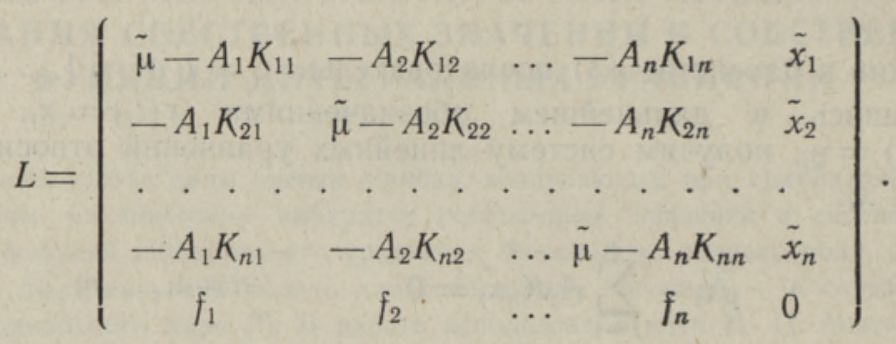

Тогда можно написать

$$
\begin{gathered}
x_{i}-\tilde{x_{i}}=\sum_{j=1}^{n} \gamma_{i j}\left[\varrho_{i}-(\mu-\tilde{\mu})\left(x_{j}-\tilde{x}_{j}\right)\right] \quad(i=1, \ldots, n), \\
\mu-\tilde{\mu}=\sum_{j=1}^{n} \gamma_{n+1, j}\left[\varrho_{j}-(\mu-\tilde{\mu})\left(x_{j}-\tilde{x}_{j}\right)\right] .
\end{gathered}
$$

Воспользовавшись оценками

$$
\begin{array}{r}
\gamma_{1} \geqslant \max _{1 \leqslant i \leqslant n} \sum_{j=1}^{n}\left|\gamma_{i j}\right|, \quad \gamma_{2} \geqslant \sum_{j=1}^{n}\left|\gamma_{n+1, i}\right|, \\
\varepsilon \geqslant \max _{0 \leqslant s \leqslant 1} \int_{0}^{1}|\varepsilon(s, t)| d t, \\
h \geqslant \max _{0 \leqslant s \leqslant 1} \sum_{j=1}^{n}\left|A_{j}\right|\left|K\left(s, t_{j}\right)\right|
\end{array}
$$

и определяя нормы

$$
\begin{aligned}
& \|x-\tilde{x}\|=\max _{1 \leqslant i \leqslant n}\left|x_{i}-\tilde{x}_{i}\right|,\|\tilde{x}\|=\max _{1 \leqslant i \leqslant n}\left|\tilde{x_{i}}\right|, \\
& \|\varrho\|=\max _{0 \leqslant s \leqslant 1}|\varrho(s)|<\frac{1}{|\mu|} \varepsilon \max _{0 \leqslant s \leqslant 1}|x(s)|,
\end{aligned}
$$
простое.

- Это равносильно предположению, что собственное значение $\tilde{\mu}$ матрицы $\left(A_{i} K_{i j}\right)$ 
приведем формулы (5) в нелинейные соотношения

$$
\|x-\tilde{x}\| \leqslant \frac{\gamma_{1}\|Q\|}{1-|\mu-\tilde{\mu}| \gamma_{1}},|\mu-\tilde{\mu}| \leqslant \frac{\gamma_{2}\|Q\|}{1-\|x-\tilde{x}\| \gamma_{2}} .
$$

Предположим, что данная запись имеет смысл. Следуя идее Э. Нистрема [5], представим собственную функцию $x(s)$ ядра $K(s, t)$ через ее значения $x_{j}$ в узлах $t_{j}$ :

$$
x(s)=\frac{1}{\mu}\left\{\sum_{j=1}^{n} A_{i} K\left(s, t_{j}\right) x_{j}+\varrho(s)\right\} .
$$

Перепишем последнее выражение еще в виде

$$
x(s)=\frac{1}{\mu}\left\{\sum_{j=1}^{n} A_{j} K_{i j}\left[\left(x_{l}-\tilde{x}_{j}\right)+\tilde{x}_{j}\right]+\varrho(s)\right\}
$$

и используем оценки (6). Тогда получим

$$
\max _{0 \leqslant s \leqslant 1}|x(s)| \leqslant \frac{h|\mu|}{|\mu|^{2}-\varepsilon}(\|\tilde{x}\|+\|x-\tilde{x}\|) .
$$

Следовательно,

$$
\|\varrho\|<\frac{h \varepsilon}{|\mu|^{2}-\varepsilon}(\|\tilde{x}\|+\|x-\tilde{x}\|) .
$$

Найденную таким образом оценку для погрешности $\varrho(s)$ подставляем в соотношения (7) и снова решаем первое неравенство (7) относительно величины $\|x-\tilde{x}\|$. Найдем, что

$$
\|x-\tilde{x}\| \leqslant \frac{\gamma_{1} h \varepsilon\|\tilde{x}\|}{\left(|\mu|^{2}-\varepsilon\right)\left(1-\| \mu-\tilde{\mu} \mid \gamma_{1}\right)-\gamma_{1} h \varepsilon} .
$$

Аналогично следует из второго неравенства (7), что

$$
|\mu-\tilde{\mu}| \leqslant \frac{\gamma_{2} h \varepsilon\|\tilde{x}\|}{\left(|\mu|^{2}-\varepsilon\right)\left(1-|\mu-\tilde{\mu}| \gamma_{1}\right)-\gamma_{1} h \varepsilon} .
$$

Итак,

$$
\|x-\tilde{x}\| \leqslant \gamma_{1} c, \quad|\mu-\tilde{\mu}| \leqslant \gamma_{2} c,
$$

где обозначено

$$
c=\frac{h \varepsilon\|\tilde{x}\|}{\left(\left.\mu\right|^{2}-\varepsilon\right)\left(1-|\mu-\tilde{\mu}| \gamma_{1}\right)-\gamma_{1} h \varepsilon} .
$$

Предположим, что $c>0$. Выбираем для $|\mu-\tilde{\mu}|$ такую оценку $l$, для которой справедливо

$$
l \geqslant \frac{\gamma_{2} h \varepsilon\|\tilde{x}\|}{\left\{(|\tilde{\mu}|-l)^{2}-\varepsilon\right\}\left(1-l \gamma_{1}\right)-\gamma_{1} h \varepsilon} .
$$


Для определения $l$ можно использовать уравнение четвертой степени

$$
\begin{gathered}
\varphi(l) \equiv \gamma_{1} l^{4}-\left(1+2 \gamma_{1}|\tilde{\mu}|\right) l^{3}+\left\{2|\tilde{\mu}|+\gamma_{1}\left(|\tilde{\mu}|^{2}-\varepsilon\right)\right\} l^{2}- \\
-\left\{|\tilde{\mu}|^{2}-\varepsilon\left(1+\gamma_{1} h\right)\right\} l+\gamma_{2} h \varepsilon\|\tilde{x}\|=0 .
\end{gathered}
$$

Этим доказана следующая

Т е орем а. Пусть ядро $K(s, t)$ имеет хоть одно собственное значение, $\sum_{=1}^{n} f_{i} \tilde{x}_{j}=1$ и существует матрица Г. Если уравнение (9) имеет положиjтельные корни и наименьший из них $l^{*}$ удовлетворяет условию

$$
l^{*}<\min \left(\frac{1}{\gamma_{1}},|\tilde{\mu}|-\sqrt{\varepsilon}\right),
$$

то для собственного значения $\mu$ ядра $K(s, t)$ и для соответствующей собственной функции $x(s)$, при которой $\sum_{j=1}^{n} f_{i} x_{i}=1$, справедливы оценки

$$
|\mu-\tilde{\mu}| \leqslant l^{*}, \quad\|x-\tilde{x}\| \leqslant \frac{\gamma_{1}}{\gamma_{2}} l \text {. }
$$

3 а меч ание 1. Нужное нам значение $l$ можно для простоты найти н из несколько более строгого условия, а именно как нанменьший положительный корень уравнения третьей степени

$$
\begin{gathered}
2 \gamma_{1}|\tilde{\mu}| l^{3}-\left\{2|\tilde{\mu}|+\gamma_{1}\left(|\tilde{\mu}|^{2}-\varepsilon\right)\right\} l^{2}+ \\
+\left\{|\tilde{\mu}|^{2}-\varepsilon\left(1+\gamma_{1} h\right)\right\} l-\gamma_{2} h \varepsilon\|\tilde{x}\|=0 .
\end{gathered}
$$

Найденное здесь значение $l$ тоже должно удовлетворять условиям (10). На практике вместо точного значения $l^{*}$ возьмем некоторое приближенное, бо́льшее значение $\tilde{l}$ (например, округленное с избытком значение $l^{*}$ ), при котором $\varphi(\tilde{l}) \leqslant 0$.

3 а мечание 2. Исходя из формул (5) и пользуясь оценками (6), можно найти, что

$$
|\mu-\tilde{\mu}| \leqslant \gamma_{2} c^{\prime}, \quad\|x-\tilde{x}\| \leqslant \gamma_{1} c^{\prime}, \quad \text { где } \quad c^{\prime}=\frac{2\|\varrho\|}{1+\sqrt{1-4 \gamma_{1} \gamma_{2}\|\varrho\|}},
$$

если только $\gamma_{1} \gamma_{2}\|\varrho\| \leqslant \frac{1}{4}$. Полученные таким образом оценки формально являются частным случаем замечания 2 одной из работ Э. Тамме [3]. Но здесь полученный результат в данном виде неприменим, поскольку величина $\|\varrho\|$ тоже неизвестна. Поэтому надо избавиться от неизвестной нам оценки $\|\varrho\|$. Легко видеть, что $\|\varrho\| \leqslant c^{\prime} \leqslant 2\|\varrho\|$. Возьмем, например, $c^{\prime}=2\|\varrho\|$ и используем неравенство (8). После несложных выкладок придем к следующему результату:

если наименьший положительный корень $k^{*}$ кубического уравнения *

$$
\psi(k) \equiv k^{3}-2|\tilde{\mu}| k^{2}+\left(|\tilde{\mu}|^{2}-d\right) k-2 \gamma_{2} h \varepsilon\|\tilde{x}\|=0
$$

* Здесь обозначено $d=\varepsilon\left(1+2 \gamma_{1} h\right)$. 
удовлетворяет условию

$$
k^{*}<\min \left(\frac{2}{\gamma_{1}},|\tilde{\mu}|-\sqrt{d}\right)
$$

то для собственного значения $\mu$ и собственной функции $x(s)$ получим более грубые (примерно в два раза), чем в (11), оценки

$$
|\mu-\tilde{\mu}| \leqslant k^{*}, \quad\|x-\tilde{x}\| \leqslant \frac{\gamma_{1}}{\gamma_{2}} k^{*} .
$$

Вместо точного значения $k^{*}$ можно опять взять некоторое приближенное значение $\tilde{k}$, при котором $\psi(\tilde{k}) \geqslant 0$.

Прим ер. Рещุим проблему о собственных значениях для уравнения

$$
\mu x(s)=\int_{0}^{1}\left(e^{s t}-\frac{t}{2}\right) x(t) d t
$$

способом механических квадратур. Для этого применим формулу Гаусса с тремя абсциссами .

Приближенное собственное значение $\tilde{\mu}$ определим из уравнения

$$
\tilde{\mu}^{3}-1,214410 \tilde{\mu}^{2}+0,141902 \tilde{\mu}-0,000492=0,
$$

откуда получим $\tilde{\mu}=1,081638$.

Для определения приближенного собственного вектора $\tilde{x}=\left(\tilde{x}_{1}, \tilde{x}_{2}, \tilde{x}_{3}\right)$ получим систему

$$
\begin{array}{r}
0,815962 \tilde{x}_{1}-0,359097 \tilde{x}_{2}-0,183756 \tilde{x}_{3}=0, \\
-0,278227 \tilde{x}_{1}+0,622071 \tilde{x}_{2}-0,309648 \tilde{x}_{3}=0, \\
-0,291339 \tilde{x}_{1}-0,581504 \tilde{x}_{2}+0,594471 \tilde{x}_{3}=0 .
\end{array}
$$

В качестве вектора $f=\left(f_{1}, f_{2}, f_{3}\right)$ примем нормированное решение сопряженной системы $f=(0,435913 ; 0,735922 ; 0,518072)$. Тогда найдем, что $\tilde{x}=(0,423028 ; 0,569883$; $0,764772)$. Обратная матрица $\Gamma$ матрицы $L$ имеет вид:

$$
\Gamma=\left(\begin{array}{rrrc}
0,818060 & -0,265954 & -0,254321 & 0,423028 \\
-0,220310 & 0,542323 & -0,282259 & 0,569883 \\
-0,375377 & -0,546593 & 0,614940 & 0,764772 \\
0,435913 & 0,735922 & 0,518072 & 0
\end{array}\right) .
$$

Погрешность $\varepsilon$ в случае второго итерированного ядра оценим формулой

$$
\varepsilon=\frac{n !^{4}}{(2 n !)^{3}} \frac{M_{2 n}}{2 n+1}
$$

где $n$ - число абсцисс и

$$
M_{l}=\max _{0 \leqslant s, t \leqslant 1}\left|\frac{\delta^{l}}{\delta \tau^{l}}\{K(s, \tau) K(\tau, t)\}\right|
$$


Поскольку сейчас $M_{l}=2^{l} e^{2}+\frac{2+l}{2} l$ и $n=3$, то $\varepsilon=2,4 \cdot 10^{-4}$. Остальные оценки (6) следующие: $\gamma_{1}=1,54 ; \gamma_{2}=1,69 ;\|\tilde{x}\|=0,77 ; \quad|\tilde{\mu}|<1,09 ; \quad h=1,47$.

Уравнения (9) и $\left(9^{\prime}\right)$ имеют соответственно вид

и

$$
1,54 l^{4}-4,35 l^{3}+4,01 l^{2}-1,188 l+4,59 \cdot 10^{-4}=0
$$

$$
3,35 l^{3}-4,01 l^{2}+1,188 l-4,59 \cdot 10^{-4}=0 .
$$

Здесь в обонх случаях можно принять $\tilde{l}=3,9 \cdot 10^{-4}$, после чего получим следующие оценки:

$$
|\mu-\tilde{\mu}| \leqslant 3,9 \cdot 10^{-4}, \quad\|x-\tilde{x}\| \leqslant 3,6 \cdot 10^{-4} .
$$

Если использовать менее точные оценки (13), то уравнение (12) имеет вид

$$
k^{3}-2,18 k^{2}+1,186 k-9,18 \cdot 10^{-4}=0 .
$$

Тогда можно принять $\tilde{k}=7,8 \cdot 10^{-4}$ и верны оценки

$$
|\mu-\tilde{\mu}| \leqslant 7,8 \cdot 10^{-4}, \quad\|x-\tilde{x}\| \leqslant 7,1 \cdot 10^{-4} .
$$

\section{ЛИТЕРАТУРА}

1. Мысовски и И. П., Вестн. ЛГУ. Сер. матем., мех. и астр., 19, 66-72 (1956).

2. Мыс ов ских И. П., Матем. сб., 48 (90), № 2, 137-148 (1959).

3. Т а м м е Э. Э., Уч. зап. Тартуск. ун-та, 102, 329-337 (1961).

4. B r a kha ge H., Num. Math., 3, H. 3, 174-179 (1961).

5. Ny ström E. J., Comment. phys.-math. Soc. Sci. Fenn., 4, 15 (1928).

Институт кибернетики Академии наук Эстонской ССР
Поступила в редакцию 1/IV 1964

\section{HEINLA}

\section{INTEGRAALVORRANDITE OMAVÅRTUSTE JA OMAFUNKTSIOONIDE LEIDMISE TAPSUSEST KVADRATUURVALEMITE ABIL}

Artiklis vaadeldakse pideva tuumaga Fredholmi teist liiki integraalvõrrandite omaväärtuste ja omafunktsioonide leidmist kvadratuurvalemite abil. Tuletatakse hinnangud (11) ning (13) vôrrandite (1) ja (4) omaväärtuste ja omavektorite erinevuse kohta.

\section{HEINLA}

\section{UBER DIE GENAUIGKEIT DER QUADRATURFORMELMETHODE ZUR BERECHNUNG VON EIGENWERTEN UND EIGENFUNKTIONEN DER INTEGRALGLEICHUNGEN}

In der Abhandlung wird die angenäherte Rechnung der Eigenwerte und Eigenfunktionen von Fredholmschen Integralgleichungen zweiter Art mit stätigem Kern nach der Quadraturformelmethode betrachtet. Es werden die Abschätzungen (11) und (13) für die Differenz zwischen den Eigenwerten und Eigenvektoren von (1) und denjenigen von (4) hergeleitet. 